\title{
Inflation of Wells score to obtain computed tomography pulmonary angiogram: an example of cognitive dissonance?
}

\author{
Authors: S Sharma, P Kelly and MB Whyte
}

\section{Aims}

With a priori knowledge of a positive D-dimer, to assess whether computed tomography pulmonary angiogram (CTPA) investigation requests to diagnose pulmonary embolism (PE) are made using an accurate Wells score.

\section{Methods}

Consecutive CTPA investigations over 2 months were analysed. At our institution, high-risk patients do not require D-dimer measurement as workup for PE. In all other patients, $\mathrm{D}$-dimer testing is requested by the emergency department and patients are subsequently referred to the medical team for consideration of further investigation. These patients are clerked with knowledge of the D-dimer result. D-dimer $>500$ $\mu \mathrm{g} / \mathrm{L}$ is reported as 'positive'. Electronic patient records (EPRs) were interrogated for the Wells score (a mandatory electronic entry for a CTPA request) and its constituent scores. Clinical notes were reviewed by an independent physician (blinded to the CTPA report) to retrospectively calculate the Wells score using only data available to the clerking physician. Wells score calculation: signs and symptoms of deep vein thrombosis $(\mathrm{DVT})=3 ; \mathrm{PE}$ as or more likely than alternative diagnosis $=3$; heart rate $>100=1.5$; immobilisation or surgery in previous 4 weeks $=1.5$; previous DVT $/ \mathrm{PE}=1.5$; haemoptysis $=1$; malignancy (on treatment, treated in last 6 months or palliative $)=1$. A Wells score $<2$ is low probability, a score of $2-6$ is moderate, and $>6$ is high. Wells scores (median, interquartile range (IQR)) were compared using a Wilcoxon signed-rank test. $\mathrm{p}<0.05$ was considered significant.

\section{Results}

145 case notes of patients with CTPAs were analysed. Twenty PEs (13.8\%) were diagnosed. Overall, 76 patients had D-dimer results prior to investigation, and 73 were positive. Of the 73 positive D-dimer results, the Wells score entered into the EPR (to obtain a CTPA request) was higher than when calculated by an independent clinician: $3.0(1.5-5.75)$ vs $2.5(0.0-3.75)$, $\mathrm{p}<0.001$. 17 of 73 patients had Wells recategorised from moderate or high risk to low risk, and all had negative CTPA.
'PE as or more likely than alternative diagnosis' was the most frequent component recoded.

\section{Conclusions}

The 'pre-test' probability (Wells score) entered by physicians as part of a CTPA request is higher than that subsequently found on independent review. The presence of a positive D-dimer result may lead to cognitive dissonance. Clinicians have an inherent bias toward action, particularly in therapeutic procedures with relatively low risk, possibly due to a personal need to show greater activism in their patient care. This is coupled with an apprehension at missing a diagnosis of PE.

Guidelines for assessment of PE should reflect the influence of clinicians' awareness of D-dimer result prior to the estimation of 'pre-test' probability.

\section{Conflict of interest statement}

None. 